\title{
A Case of Duodenal Tumor Adjacent to the Diverticulum That Was Resected by the Technique of Partial Submucosal Injection Combined with Underwater Endoscopic Mucosal Resection
}

\author{
Yusaku Takatori Motohiko Kato Atsushi Nakayama Naohisa Yahagi \\ Division of Research and Development for Minimally Invasive Treatment, Cancer Center, Keio University School of \\ Medicine, Tokyo, Japan
}

\section{Keywords}

Duodenal tumor · Diverticulum · Partial submucosal

injection · Underwater endoscopic mucosal resection

\begin{abstract}
A 66-year-old male was referred to our hospital for treatment of duodenal tumor. The most difficult part was that the lesion was adjacent to duodenal diverticulum. Endoscopic mucosal resection (EMR) was difficult because submucosal injection spread broadly and quickly and makes it difficult to visualize the diverticulum edge of the lesion. Simple underwater EMR (UEMR) had risk for perforation at the diverticulum part because duodenal diverticulum is spurious diverticulum that defects the proper muscle layer. Therefore, to make sufficient distance between diverticulum and the lesion, we performed partial submucosal injection into only the diverticulum side of the lesion combined with simple UEMR. The lesion was resected en bloc without any adverse events. Histopathological diagnosis was tubular adenoma with moderate atypia and surgical margin negative. Partial submucosal injection combined with simple UEMR might be useful for duodenal tumor that has any technical difficulties as in this case.

(c) 2020 The Author(s)

Published by S. Karger AG, Basel
\end{abstract}

karger@karger.com

www.karger.com/ddi

Karger ${ }^{\prime \prime}=$

BOPEN ACCESS
(C) 2020 The Author(s)

Published by S. Karger AG, Basel

This article is licensed under the Creative Commons AttributionNonCommercial-NoDerivatives 4.0 International License (CC BYNC-ND) (http://www.karger.com/Services/OpenAccessLicense) Usage and distribution for commercial purposes as well as any distribution of modified material requires written permission.

\section{Introduction}

Endoscopic resection for duodenal tumor is considered as technically difficult and at high risk of adverse events such as perforation when compared to that for the other GI tract because of poor maneuverability of the scope and thin duodenal wall. Duodenal diverticulum is second most frequent in digestive tract following colon and spurious diverticulum that defects proper muscle layer. Taken together, endoscopic resection for duodenal tumor adjacent to duodenal diverticulum is very difficult and has high risk for perforation. However, there is no report about endoscopic resection for duodenal tumor adjacent to diverticulum. Here, we report a case of duodenal tumor adjacent to duodenal diverticulum successfully resected by the technique of partial submucosal injection combined with simple underwater endoscopic mucosal resection (UEMR).

\section{Case Presentation}

A 66-year-old man was referred to our hospital for treatment of duodenal tumor. In preoperative check endoscopy, the lesion was observed at the outside wall in the descending part of the duodenum. The oral side of the lesion was adjacent to duodenal 


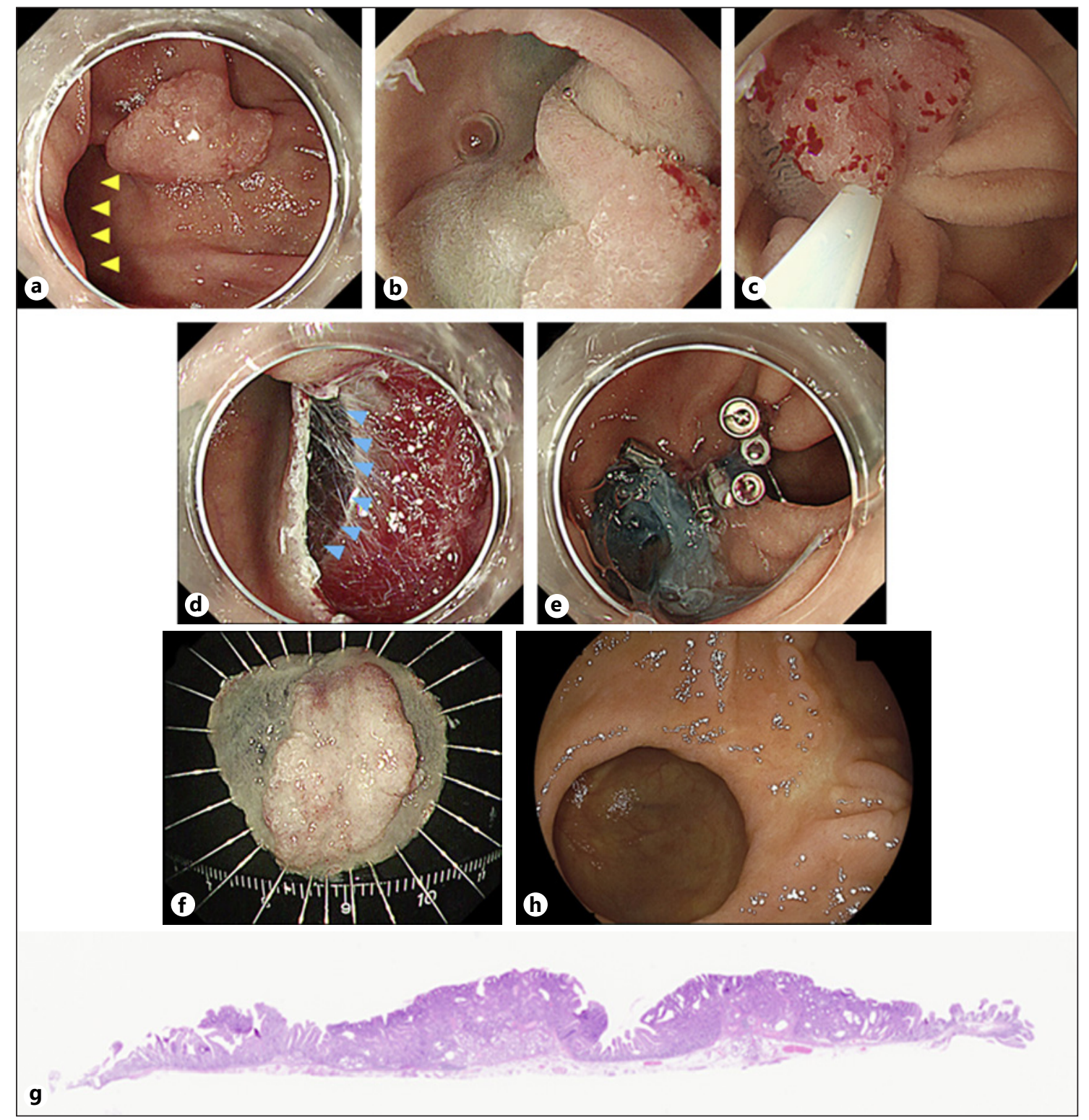

Fig. 1. Highlights of this procedure. a The target lesion was adjacent to the duodenal diverticulum. b Partial submucosal injection of sodium hyaluronate into the diverticulum side of the lesion. c Endoscopic image showing the target lesion snared. $\mathbf{d}$ We could resect the lesion without remnant and perforation. Furthermore, we found a clear defect of muscular layer through the submucosal

diverticulum, and it was the most difficult part for endoscopic resection (Fig. 1a). There were no significant signs that indicate malignancy with white light image and magnified image-enhanced endoscopy. Endoscopic mucosal resection (EMR) might be difficult because injected solution would disturb visualization of duodenal edge of the lesion. And submucosal injection solution quickly spread broadly that would make it difficult to capture the lesion with snare. Simple UEMR had a risk of perforation in the diverticulum side of the lesion because duodenal diverticulum de- layer in the diverticulum side of the resection wound (blue arrow head). e The resection wound was closed with an endoclip and covered partially with a polyglycolic acid sheet. f, $\mathbf{g}$ Resected specimen. The histopathological diagnosis was tubular adenoma with moderate atypia, and the histopathological margin was negative. $\mathbf{h}$ There was no recurrence in follow-up endoscopy after 6 months.

fects the proper muscle layer. Therefore, we planned partial submucosal injection into only the diverticulum side of the lesion before performing UEMR to make sufficient margin between the lesion and diverticulum (Video 1). For this procedure, we used a high-vision gastroscope with a waterjet function (GIF-H290T; Olympus, Tokyo, Japan) and an electro-current unit (VIO3, ERBE Electromedizin, Tuebingen, Germany). The setting of electro-current unit was Endo cut Q, effect 1.0. Oval-type endoscopic snare (Snaremaster [15 mm], Olympus, Tokyo, Japan) was cho- 


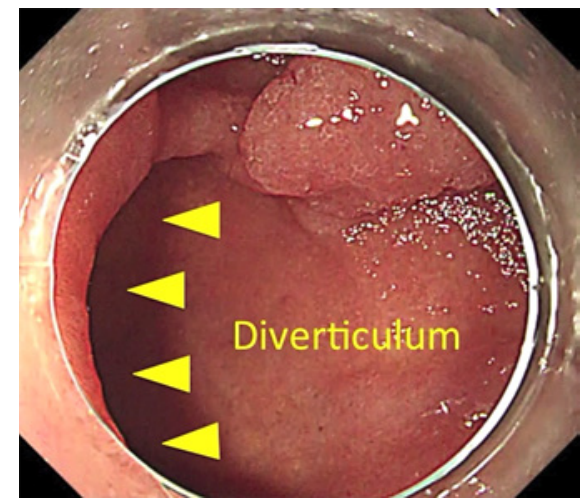

Video 1. The video highlights the partial submucosal injection technique combined with simple UEMR. UEMR, underwater endoscopic mucosal resection

sen. At first, we filled the lumen of duodenum with warmed normal saline after evacuating air. Then we injected only $0.2 \mathrm{~mL}$ of sodium hyaluronate into the submucosal layer in the diverticulum side of the lesion (Fig. 1b). After that, we promptly captured the lesion with recognizing distal side of endoscopic snare and sufficient margin (Fig. 1c). Finally, we resected the lesion without remnant and any adverse events. Furthermore, we found a clear defect of proper muscle layer, not perforation, due to spurious diverticulum through the submucosal layer in the resection wound (Fig. 1d). Although we closed most part of the resection wound with endoclips to avoid exposure bile acid and pancreatic juice, it was impossible to close completely because the space of diverticulum side was too cramped and maneuverability of an endoscope was limited. And we also covered this part with polyglycolic acid sheet, where proper muscle layer is lacking (Fig. 1e). Histopathological diagnosis was tubular adenoma with moderate atypia, and surgical margin negative (Fig. 1f, g). Clinical course of the patient was good and there was no recurrence in follow-up endoscopy after 6 months (Fig. 1h).

\section{Discussion}

Duodenal diverticulum was first described by Chomel in 1710 and is the second common following the colonic diverticulum $[1,2]$. It was reported that the prevalence of duodenal diverticulum ranges from 5 to $32 \%$ [3]. It often locates the second part of duodenum and very close to the main duodenal papilla [3]. And duodenal diverticulum is known as spurious diverticulum, lacking of proper muscle layer. Generally, endoscopic treatment for duodenal tumor is reported to be at high risk of adverse events due to anatomical features such as thin duodenal wall and poor scope maneuverability brought by angulation of the duodenum [4]. Another point is that it is technically difficult because only a little excessive injection easily makes it impossible to capture the target lesion with snare forceps, since injected solution would spread stereoscopically, not homogeneously. Taken together, endoscopic resection for lesion adjacent to duodenal diverticulum is considered as very risky for perforation.

Shichijo et al. [5] reported a case of simple UEMR for colorectal lesion surrounded by diverticula. Simple UEMR was also one of the options as in the case report. However, in this case, it was difficult to visualize completely the edge of the lesion in diverticulum part from the endoscopic field of view during procedure. This fact had the risk of unaccomplishment of complete resection even in underwater condition. Furthermore, especially this case needed to achieve en bloc resection securely because additional endoscopic treatment for recurrence lesion was considered very difficult. Siau et al. [6] reported feasibility of simple UEMR for colorectal lesion and some effectiveness of submucosal injection combining simple UEMR for difficult situations. However, submucosal injection for duodenum would spread broadly followed by difficult snaring as we mentioned above. Therefore, we decided simple UEMR was not the appropriate treatment for this case and decided to inject only partially into the area adjacent to the diverticulum to keep surgical margin as well as safety margin between the lesion and diverticulum, in combination with filling natural saline inside duodenal lumen. In this case, we used sodium hyaluronate for submucosal injection agent because the solution has high viscosity that can keep the formation of mucosal elevation for a long time compared to other agents. And the other technical tip was that we started snaring promptly just after partial submucosal injection because mucosal elevation in the duodenum spread broadly in a relatively short time even if we used sodium hyaluronate. The concept and schematic diagram of this technique is shown in Figure 2. Partial submucosal injection makes safety margin between the lesion and diverticulum. In fact, we could observe remnant of blue dined sodium hyaluronate partially in the submucosal layer of the resected wound (Fig. 1d, blue arrow head). And we could observe also muscular defect, not perforation, in diverticulum part through remnant sodium hyaluronate in the submucosal layer. Moreover, to keep safety in the opposite side of diverticulum, we used floating force by filling natural saline inside the duodenal lumen just like the simple UEMR method. It enabled to capture the lesion excluding muscular layer. Finally, we achieved en bloc resection without perforation using this optional technique and did not observe local recurrence after 6 months. 
Fig. 2. Schematic diagram of this technique. a The target lesion was adjacent to the diverticulum. b Normal saline immersion. c Submucosal injection of sodium hyaluronate in the diverticulum side of the lesion. d Capture and resection of the lesion with sufficient margin. e The lesion was resected without remnant and complication.

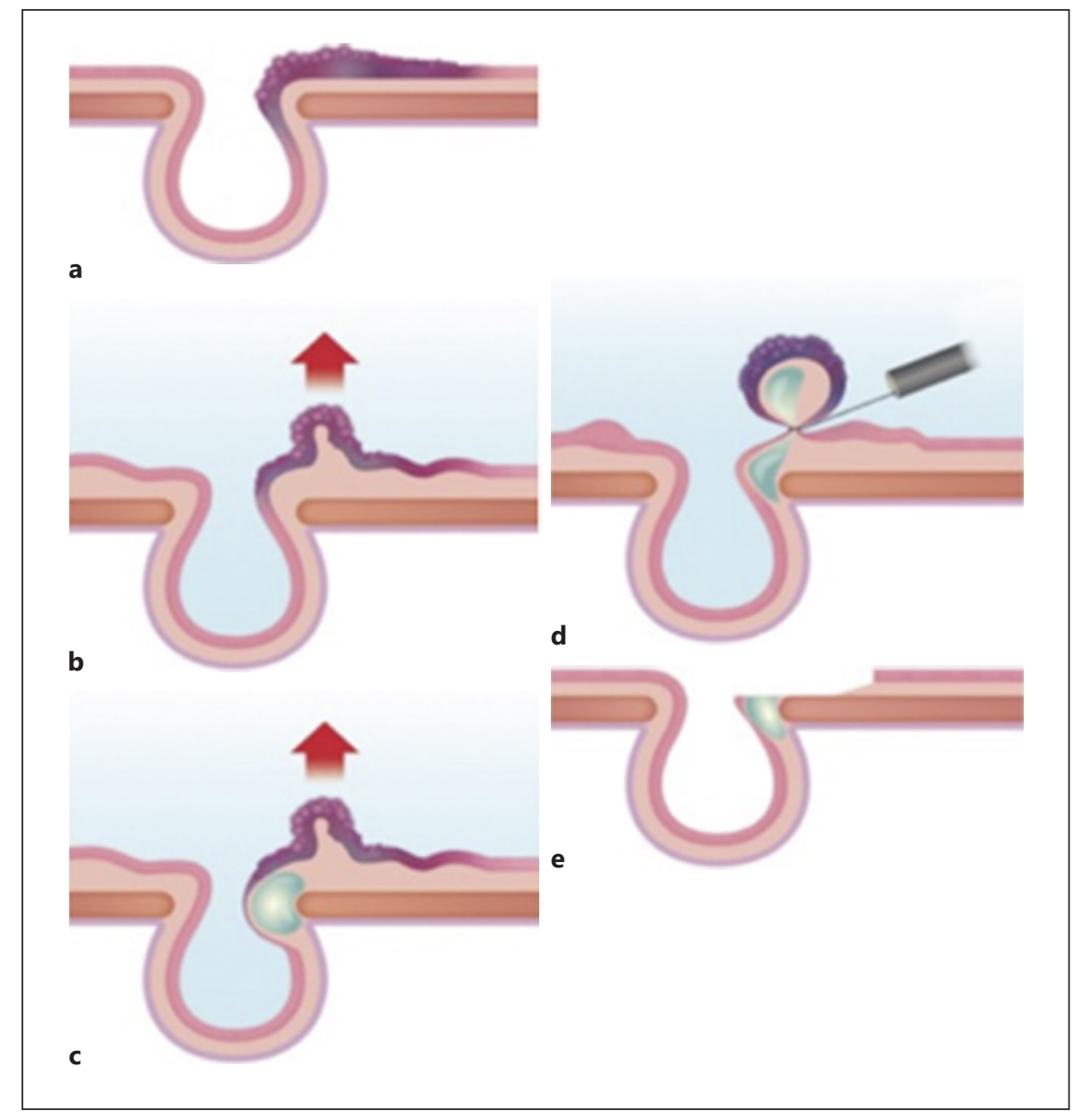

Partial injection combined with simple UEMR would be useful for other difficult lesions as well as lesions involving diverticulum. The strategy of endoscopic treatment for duodenal tumor has been controversial because of insufficient evidence. It has been reported that simple UEMR using floating force underwater condition is useful and safe even for duodenal tumor with non-lifting sign $[7,8]$. However, we reported that en bloc resection rate of UEMR is significantly lower than EMR for duodenal tumor [9]. The lesions that are located at the inside wall of the duodenal angle or relatively large lesions make it difficult to achieve en bloc resection with simple UEMR as well as in this case because neither of them can visualize the distal edge of the lesion. Partial injection combined with simple UEMR would be better because we could obtain better visualization of the distal edge of the lesion. If this technique is feasible for them, it is expected that more cases will be treatable with this technique instead of ESD that has high risk for complication. Future study is requested to confirm technical feasibility and safety of this technique.

\section{Statement of Ethics}

The patient gave written informed consent to publish the case.

\section{Conflict of Interest Statement}

The authors have no conflicts of interest to declare.

\section{Funding Sources}

The authors did not receive any funding.

\section{Author Contributions}

Yusaku Takatori was the main operator of this case and contributed to this manuscript. Motohiko Kato designed the concept of this procedure. Atsushi Nakayama contributed to editing the video. Naohisa Yahagi approved the final draft. 


\section{References}

1 Pearl MS, Hill MC, Zeman RK. CT findings in duodenal diverticulitis. AJR Am J Roentgenol. 2006;187(4):W392-5.

2 Costa Simões V, Santos B, Magalhães S, Faria G, Sousa Silva D, Davide J. Perforated duodenal diverticulum: surgical treatment and literature review. Int J Surg Case Rep. 2014;5(8): 547-50.

3 Jakubczyk E, Pazurek M, Mokrowiecka A, Wozniak B, Malecka-Panas E, Podgorski M, et al. The position of a duodenal diverticulum in the area of the major duodenal papilla and its potential clinical implications. Folia Morphol. 2020.
4 Yamasaki Y, Uedo N, Takeuchi Y, Ishihara R, Okada H, Iishi H. Current status of endoscopic resection for superficial nonampullary duodenal epithelial tumors. Digestion. 2018; 97(1):45-51.

5 Shichijo S, Yamaguchi Y, Nakahara M, Imai Y, Ishihara R. Underwater EMR of a colonic adenoma surrounded by diverticula. VideoGIE. 2020;5(4):157-8.

6 Siau K, Ishaq S, Cadoni S, Kuwai T, Yusuf A, Suzuki N. Feasibility and outcomes of underwater endoscopic mucosal resection for $\geq 10$ mm colorectal polyps. Surg Endosc. 2018;32: 2656-63.

7 Shibukawa G, Irisawa A, Sato A, Abe Y, Yamabe A, Arakawa N, et al. Endoscopic mucosal resection performed underwater for nonampullary duodenal epithelial tumor: evaluation of feasibility and safety. Gastroenterol Res Pract. 2018;2018:7490961.
8 Binmoeller KF, Shah JN, Bhat YM, Kane SD "Underwater" EMR of sporadic laterally spreading nonampullary duodenal adenomas (with video). Gastrointest Endosc. 2013; 78(3):496-502.

9 Kiguchi Y, Kato M, Nakayama A, Sasaki M, Mizutani M, Tsutsumi K, et al. Feasibility study comparing underwater endoscopic mucosal resection and conventional endoscopic mucosal resection for superficial non-ampullary duodenal epithelial tumor $<20 \mathrm{~mm}$. Dig Endosc. 2019. 